This item was submitted to Loughborough's Research Repository by the author.

Items in Figshare are protected by copyright, with all rights reserved, unless otherwise indicated.

\title{
Exploring the social and organisational aspects of human factors integration:
}

\section{a framework and case study}

PLEASE CITE THE PUBLISHED VERSION

PUBLISHER

(C) Elsevier

VERSION

AM (Accepted Manuscript)

LICENCE

CC BY-NC-ND 4.0

\section{REPOSITORY RECORD}

Waterson, Patrick, and Stephven Lemalu-Kolose. 2019. "Exploring the Social and Organisational Aspects of Human Factors Integration: A Framework and Case Study". figshare. https://hdl.handle.net/2134/5957. 
This item was submitted to Loughborough's Institutional Repository (https://dspace.lboro.ac.uk/) by the author and is made available under the following Creative Commons Licence conditions.

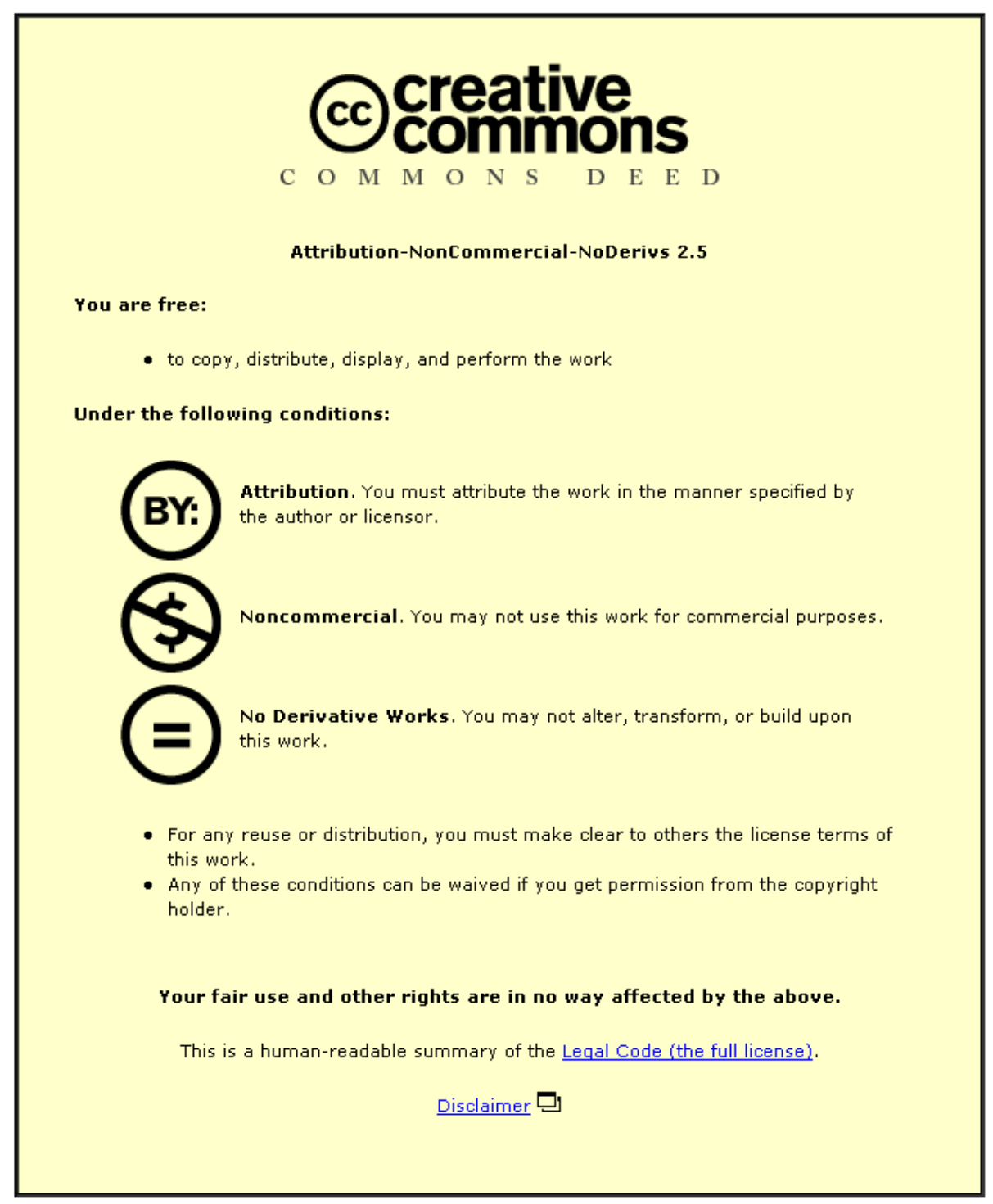

For the full text of this licence, please go to: http://creativecommons.org/licenses/by-nc-nd/2.5/ 


\title{
Exploring the Social and Organisational Aspects of Human Factors Integration:
}

A Framework and Case Study

\author{
Patrick Waterson ${ }^{1}$ and Stephven Lemalu Kolose ${ }^{2}$ \\ ${ }^{1}$ Department of Ergonomics \\ Loughborough University \\ Loughborough, LE11 3TU, \\ United Kingdom \\ ${ }^{2}$ Human Systems Group \\ Defence Technology Agency (DTA) \\ New Zealand Defence Force \\ Private Bag 32901 \\ Devonport, Auckland \\ New Zealand
}

Corresponding author:

Dr. Patrick Waterson

Department of Ergonomics

Loughborough University

Loughborough, LE11 3TU, United Kingdom.

Tel: 01509228478

Fax: 01509223940

Email: p.waterson@lboro.ac.uk 


\title{
Exploring the Social and Organisational Aspects of Human Factors Integration: A Framework and Case Study
}

\begin{abstract}
In this paper we first outline a framework which aims to capture some of the social and organisational aspects of Human Factors Integration (HFI) which have been outlined by previous research. The framework was partly used to design a set of interview questions that were used with a case study of a human factors team working with the UK defence industry. The findings from the case study revealed a number of barriers which accord with previous research in the domain of HFI (e.g., attitudes and perceptions towards $\mathrm{HF}$ ), as well as providing insights into the improvement strategies used by the HF team in order to improve HFI. These included attempts to build relationships and establish a working rapport with other groups in the company, as well as other activities aimed at addressing the organisational culture within the company as a whole (e.g., attempts to raise the profile of HF within the company). We use the framework for social and organisational aspects of HFI to discuss our findings alongside other research on group behaviour and boundary management within large organisations. The conclusions of the paper point to the utility of the framework as a means of planning HFI improvement strategies which can help to overcome some of the social and organisational barriers to HFI.
\end{abstract}

\section{Keywords}

Human Factors Integration; social and organisational aspects of Human Factors; Human Factors barriers and enablers. 


\section{Introduction}

Accounts of the problems involved in applying human factors within industry have a long history and date back to some of the earliest examples of research and practice within human factors and ergonomics (Meister, 1999; Chapanis, 1999; Waterson and Sell, 2006). Over the years a number of authors have pointed to a range of problems and barriers that underpin the relationship between human factors practitioners and their industrial counterparts. In particular, the work of David Meister and colleagues during the 1960's, 70's and 80's has addressed these issues in depth. Meister and Farr (1967) for example, found that designers and engineers had little or no interest in human factors, partly since human factors information was perceived as being too inaccessible as compared to charts, graphs and tables. Later work during the 1970s and early 1980s (Meister 1982a,b) sought out the views of engineers, research contractors and government personnel regarding human factors. One of the main conclusions from this research that individuals were not convinced of the value of human factors and were inadequately trained in the use of HF tools and methods.

In his analysis of the influence of organisational context on the work of human factors engineers (HFEs), Charles Perrow (1983) argued that the relative weak position and low profile of the HFE within the context of the larger organisation in which they are employed, served to undermine the value and impact of their work. Various other explanations for this type of phenomenon can be given, including the differences in terms of "mindsets" and values which exist between HFEs and colleagues drawn from other disciplines and backgrounds (e.g., social science as compared to engineering Cullen, 2007). In addition, many have pointed to the high costs of inputs from human factors into the design process and HF assessments and evaluations (Beevis, 2003; Kerr et al., 2008).

\subsection{Human Factors Integration (HFI)}

In the last few years the drive toward improving the uptake of human factors within industry has led to the development of initiatives to support human factors integration (HFI). Newman and Tatlock (2004) report the outcomes of a workshop held with HFI practitioners from industry in order to ascertain the types of barriers that exist when applying HFI within system design. The workshop generated over 190 recommendations and covered a range of issues including the provision of "help 
desk" facilities to help manage the HFI process, the need for better structured tools and methods for the identification of HFI issues and methods for quantifying the costs and benefits of HFI. Similarly, the report highlighted the importance of organisational factors such as culture and attitudes amongst system designers in shaping the outcomes of HFI initiatives.

Much of the research that has been carried out on HFI has focused on the development of method and tool development (Newman et al., 2008; MacLeod, 2008). Similarly, others have focused on the need to develop cogent arguments for cost justification of HFI (Bruseberg, 2008). Little recent work has concentrated in detail on the social and organisational "process issues”, namely: the day-to-day work of HF teams; their interaction with other groups; communication problems and barriers; and, the influence of large-scale extrinsic factors such as organisational culture.

In the next section of the paper we describe the framework for understanding the social and organisational aspects of HFI. The framework brings together previous research on the organisational constraints on HFI (e.g., Perrow, 1983), as well as other research which has examined the components of effective team working within organisations (e.g., Antoni and Hertel, 2009; Sundstrom, DeMeuse and Futrell, 1990). The framework was partly used to design an interview schedule that was used with a case study of the social and organisational aspects of HFI (sections 3 and 4). In the discussion section of the paper (section 5) we use the framework to shed further light on the findings from the case study, as well as considering the wider implications of these findings for future research on HFI.

\section{A framework for understanding the social and organisational aspects of HFI}

The work of human factors teams within organisations can be seen as being influenced by a range of factors which cut across organisational, team and individual levels of analysis. At the wider organisational level, reward systems, the structural characteristics of the organisation and it's cultural values will play a role in how they operate (Schein, 2005). We know from previous work on HFI for example, that the work of the HF team is often marginalised and their role in the business of the organisation may be seen as unimportant or peripheral (Perrow, 1983). 
The effectiveness with which they function as a team within the organisation is sometimes seen as consisting of two main dimensions: team performance and team viability (Hackman, 1987; Sundstrom, DeMeuse and Futrell, 1990). Team performance refers to the degree to which the work team meets standards given by supervisors or customers within or outside the wider organisation. In the context of a HF team this might mean the degree to which the team is seen as providing support to the design process or supporting the large business goals within the organisation. Team viability refers to extent that collaboration between members of the team an other groups within the organisation is sustained over time. In this case, this might refer to how likely the involvement of the HF team in projects is likely to extend beyond short-term project work and progress into longer-term collaborations within the organisation.

Finally, at an individual level of analysis attitudes and mindsets regarding the value of $\mathrm{HF}$ and its role in the design progress are likely to shape the way in which the HF team work within the organisation (Meister 1982 a, b). Figure 1 shows a diagram of how these organisational, team and individual levels of analysis relate to one another, alongside examples of social and organisational barriers preventing effective HFI.

Figure 1 about here

\subsection{Study aims and objectives}

The framework is used in part to probe deeper into the social and organisational aspects of HFI using a case study of a human factors team working within an industrial, defence-based context. Specifically, the aims of the paper are threefold: 
- To examine in detail an industrial case study of the social and organisational processes that shape the work of a human factors team and their relationship with colleagues from other disciplines;

- To describe the main barriers, problems and enables of the work of the human factors team and compare this with previous research on HFI.

- To interpret the findings from the case study in terms of the social and organisational aspects of HFI framework described earlier.

\section{Method}

\subsection{Setting and team characteristics}

The study took place within a division of a large engineering company based within the UK. The main business of the company division is taken up with defence applications and the application of human factors design assessment and evaluation techniques. The human factors team work in close partnership with contractors from the defence industry, as well as engineers and other personnel responsible for safety employed by the company. The team is comprised of approximately 8 members and is well established within the company, having been in existence for over ten or so years. Membership of the team is relatively stable and their role in the company is clearly defined in terms of operational procedures. The activities of the human factors team corresponds to the definition of a "work team” as described by Cohen and Bailey (1987), in that they are charged with performing tasks associated with the core activities of the company (e.g., evaluating the safety of defence equipment and putting forward suggestions for improvements to design). The work of team often involves a small number of the 8 members working on project work. In other cases the team may be tasked as a whole to work on longer-term project work. Due to the confidential nature of the work conducted by the team we have disguised references to specific projects, as well as other details of their work which would have compromised their anonymity.

\subsection{Participants and recruitment}


A total of 20 interviews were carried out during June and July 2007. Five participants were members of the human factors team: six were employed as engineering designers; six participants worked as safety engineers. The remaining three participants worked as engineers within the company with varying responsibilities, company experience and roles (e.g., Head of Engineering Division) - table 1.

Table 1 here

A list of potential participants for the study was provided by the Human Factors Team Leader and subsequently contacted by one of the researchers by email in order to arrange dates and times for interview. A purposive sampling strategy was used in order to ensure that a representative sample of company roles (e.g., managers and team leaders) and disciplines took part in the study (e.g., human factors team members, design engineers). The interviews lasted between 30 minutes to 1 hour. In addition, a number of participants were later contacted by email in order to clarify material derived from the interview, as well as to give them the opportunity for further comments.

\subsection{Interview schedule}

A semi-structured interview schedule was drawn up and based upon the findings from previous research examining the barriers and enablers to successful human factors integration. In this context we define a barrier as an "obstacle or agency that prevents the integration of HF into project work". The term enabler, by contrast we take to refer to "something that eases or supplies the means to bring about the integration of HF into project work". Both definitions are based on those provided by the most recent edition of the Oxford English Dictionary (11 ${ }^{\text {th }}$ edition, 2008). A draft of the schedule was piloted with members of the human factors team. The final interview schedule was made up of the following sections: the professional background of the interviewee; experiences with human factors and human factors techniques; opinion of the role of human factors in the design process; experiences of 
successful/unsuccessful human factors integration; reasons why the company may not involve human factors in design; the role played by human factors more generally within the company and attitudes within the company towards human factors; and, future challenges for human factors within the company and suggestions how these could be improved or facilitated.

\subsection{Data Analysis}

All of the interviews were recorded using a digital recorder and manually transcribed into Microsoft Word documents. The data was then inputted into the NVivo (version 7) qualitative data analysis software package. Template analysis (King, 1998; Crabtree and Miller, 1999) was used to further examine and develop codes using a set of a priori codes based upon data within the headings and sub-heading in the interview schedule. Once the a priori codes had been defined they were subsequently applied to the whole dataset. During the second phase of data analysis the original codes were reanalysed and a number of additional themes and sub-themes emerged from the data. This process followed the guidelines outlined by Miles and Huberman (1994) and resulted in a final coding scheme (table 2).

Table 2 here

\section{Results}

4.1 The work and role of the human factors team

Much of the work of the human factors team is mandatory, in the sense that projects are required to have human factors input due to legal and safety considerations:

"There is a split in our human factors integration ... You must have human factors input, which is good because obviously everybody in the project knows that human factors integration must happen" (Human Factors Team Member) 
In other cases there is a degree of flexibility, whereby the team, may be called into projects on an informal basis and their opinion sought on a variety of issues relating to human factors:

"It could be anything from a couple of hours to attend a meeting to providing human factors or specialist expertise, right the way through to a year to two-year project ... (Human Factors Team Leader)

A distinction can be drawn between examples of the work of the team where they have been "pulled" into projects through an external request and other instances where the team actively "pushes" themselves into projects. Frequent examples of the "pull" aspects of the work of the team involve information provision, often in the form of anthropometric, human reliability and other forms of HF data:

"Within our organisation the human factors team are kind of data suppliers to our safety work. We carry out a risk model which obviously has questions based on the user. We ask for the information from the human factors team, which is generally human reliability assessment data” (Safety Engineer)

The team also actively attempts to "push" HF into design and raise the awareness of other project members of the need to consider HF when carrying out design activities:

"It could be sometimes that we suggest someone has human factors. For example, [posing a rhetorical question] do you realise that there is a lot of human factors in there? Sometimes the external customer will then contact us." (Human Factors Team Leader)

This strategy has met with some success and has led to a growing awareness of the work of the team and the benefits of their involvement:

"People will request your help, invariably because they have got some awareness of ergonomics and human factors or they have seen the project where human factors and ergonomics input has had some positive effect and are not necessarily aware of what that input is and where it should come from, or the nature of the activity involved." (Human Factors Team Member)

External perceptions of the role of team varied across a few dimensions, most comments viewed the team as a fundamental part of the business activity of the 
company. Part of this was interpreted as maintaining quality in terms of safety compliance or risk mitigation:

"It ensures that ... you don't have the risk of having to do potentially significant rework because the delivered product isn't what the customer was looking for ... getting HF involved is to some extent derisking the eventual delivery." (Safety Engineer)

The comments of the Head of Engineering reinforce the view that human factors is perceived as part of the core business of the company. The human factors team are not seen as separate, or peripheral to engineering, but rather as an integrated component:

"It's more to do with implementing systems standards, rather than analyzing a human factors situation...In [the company] we treat human factors as just another engineering skill.” (Head of Engineering)

\subsection{Social and organisational factors influencing HFI}

\subsubsection{Company culture}

The culture within the company was seen as one of the factors that acted as an impediment to the work of the human factors team. Part of this related to communication problems:

"Why organizations might not use or utilise human factors? - It would be down to lack of understanding, a lack of communication or perception, that it is too expensive or too time-consuming. (Human Factors Team Member)

In other cases there existed a shared lack of understanding of what was covered by the work of the team:

"I really think most managers don't understand it, some engineers do, but not many." (Head of Engineering)

An additional problem was that although the human factors team had been in existence for a number of years and had an established track record within the company, it still lacked visibility and prominence within the overall organizational structure: 
"I think HF is always seen as the sort of fluffy bolt on part of the project design. It's relatively new in that its been around for many years ...it can sometimes be seen as a bubble on a flow diagram of an organisational chart." (Safety Engineer)

The lack of high profile of the team acts as a further barrier in terms of integrating their work within engineering, as well as extending the scope of their work into new areas:

“... a major lesson learned is to get HF personnel integrated ... Is that a shared view across the organisation? ... I think not, I think that there are a number of project managers and senior engineers out there who understand probably the same position as myself, but I think there is another group who may not even know that they exist to be honest." (Safety Engineer).

\subsubsection{Relationships}

Respondents frequently mentioned the nature of relationships between the human factors team and their colleagues as one of the most important factors in achieving successful HFI in projects. Building a relationship and maintaining good relations with project members initially involved getting over skepticism and to some extent mistrust of the value of HF:

"It's nice to see in the beginning when people ask "what are you all about?" By the time you've explained it to them and done the projects, they can see the benefits. Now they seek human factors guidance rather than being forced to." (Human Factors Team Member)

Building the relationship took time and over the course of the last few years attitudes towards the work of the team have improved:

"Initially, everyone thought that it was a complete waste of time because they didn't see the point of it. But obviously, as time goes on you certainly see the benefits." (Safety Engineer) 
Improved relationships have evolved to the extent that HF involvement and the benefits of making use of the expertise of the team, has been established amongst many people as a norm within the Company:

"It's very clear that human factors is a major part of our business. This has only really risen up over the last three to five years, and I think it is was there, but was a little bit dormant. Nowadays, it is recognized" (Safety Engineer).

In some cases, respondents expressed the opinion that whole-scale integration of HF activities with engineering and design had been achieved:

"There is really no difference in what we do and what the human factors people provide. We are all one team essentially, whatever engineer you are, we are all the same." (Safety Engineer)

Establishing a rapport with other disciplines outside of HF and building on this during project work, involved a variety of actions on the part of the human factors team. These included being proactive, supportive and maintaining a positive attitude toward working in multidisciplinary teams:

"One of the biggest things that I've learnt about human factors integration here is that you have to work with other disciplines, work effectively with other disciplines for the human factors integration part ... build up on that rapport and the movement of information, being clear on what the software engineer or the hardware engineer are trying to achieve." (Human factors Team Member)

In some cases it might also mean trying to understand the needs of other colleagues and adopting their point of view:

"It is basically important to understand the context of the project and try to go on from there and be quite proactive. If you show enthusiasm and pump a few more ideas into the process ... they will be quite forthcoming with you. It's also about building a rapport as well” (Human Factors Team Member)

Maintaining the relationship also required persistence and determination: 
"I've seen some thorough hard work by the human factors people over the years and they demonstrate and provide valuable input, but it's not necessarily a natural joining together of the technical people and the actual human factors people. It has to continually be propagated by the human factors side" (Safety Engineer).

The friendliness of the team and approachability was singled out as reasons underpinning successful relationships and helped to cement these together:

"The human factors team are very, very friendly people and easy to know. I think people like [name] must have the patience of a saint to have the courage to run human factors and handle the criticisms and grief from the other side." (Safety Engineer)

The approachability of the team acted as a way of opening up channels of communication:

"The HF people are very open approachable lot, they were forthcoming with the answers and there's a lot of two way communication." (Design Engineer)

\subsubsection{Learning}

One of the processes that has led to greater integration of HF within engineering projects has been through mutual learning. Human factors team members and colleagues from other disciplines have learnt to appreciate and value their different roles and viewpoints on design issues:

"I think it was basically a learning process from both sides, the design engineer's understanding what the human factors people had to do, and likewise the human factors people understanding what the designers had to do." (Development Engineer)

Part of this mutual learning has involved appreciation of the differences that exist between the goals and viewpoints of human factors team members and other disciplines:

"There's a mixed aspect to the relationship, I think its positive in that human factors drives towards having a better design, and I think the negativity comes from where human factors obviously wants to do a full job when they look at things. They want to be quite thorough, whereas the management of the design team probably want to get 
a maximum return of what they can get but in a small space of time and money so they aren't using too much budget." (Design Engineer)

"I think it's quite a good attitude now, there's none of this 'here we go human factors is now coming in to stop us'. I also think that as a designer, we might not always appreciate the environment that the operator works in, so human factors can be our saviour at times." (Design Engineer)

\subsubsection{Communication and mutual understanding}

Communication and problems of communication were mentioned by respondents as factors that determined the success of HF integration within the company. In some cases, respondents pointed to difficulties in understanding as a barrier to integration:

"They absolutely need to understand what engineers are talking about, so that they do need the basic man-machine interface training and a basic engineering understanding of how things work, because you just can't get on at all otherwise”. (Safety Engineer)

Respondents sometimes referred to styles and modes of communication as ways of improving integration:

"They should have a welcoming disposition towards them, as they have some valuable ideas to provide” (Domain Engineer)

In some cases the ability of an individual to put over a point of view, particularly with managers was seen as advantageous:

"it's good communication with the people that manage the project, and amongst different teams that were taking part in the project, and then really after that it often comes down to individuals as to how well it flows" (HF Subcontractor Safety Engineer)

The problems involved in the nature of HF data, finding the right level of detail, as well as the difficulties in providing an unequivocal opinion relating to HF data were also mentioned as sometimes leading to communication problems

"What you do find is that at a level of detail you get a lot of conflicting advice between various human factors experts, not even that, [it's] more the priority or importance of an aspect." (Safety Engineer) 
Presenting data in a particular format was seen as facilitating translation across disciplinary boundaries:

"If it's something looking at a manual handling issue, then you need a pictorial sort of way to present that to the engineers ... and that's when it adds value." (Human Factors Team Leader)

\subsection{HFI Barriers}

\subsubsection{Attitudes towards HF}

The view that human factors was "common sense" was mentioned by a number of respondents as a significant barrier in establishing HF within projects:

"Sometimes you don't feel valued because you are seen as common sense or an add on and some people are not open-minded enough to see that you can add value to their project." (Human Factors Team Leader)

In other cases, even where there was established support and enthusiasm for the work of the HF team, the view that HF was "just common sense” was still present amongst those outside of the team:

"We used a human factors approach, using our expert to say, come on this is common sense, and in some respects this is the problem with human factors isn't it? A lot of it is common sense, and a lot of that is quite clever in the design analysis technique. But some of it is common sense" (Head of Engineering)

Another attitude that seemed to be common was that HF was a "tick box" activity, which should be carried out quickly and with a minimal of disruption:

"You might have to design something that is almost ready to manufacture but then think 'oh dear, I've got to tick the human factors box', human factors will take a quick look at the design and then have quite a detailed look at the tool which could be quite useful, but from the designer's point of view, a waste of time as it also increases the time of the design process without even having any added benefit." (Design Engineer)

4.3.2 Knowledge and experience: engineering and human factors 
Some respondents mentioned that there were occasions when there were tensions between engineers and the human factors team with regard to HF knowledge and expertise:

"You get an attitude of 'I did a module of this when I did systems engineering at university eight years ago' so therefore, I know what I'm talking about, I'm not letting some human factors engineer tell me what my products or system should or shouldn't be.” (Human Factors Team Member)

Likewise, there was also the view that the human factors team need more engineering knowledge and experience in order to work within projects:

"I think the human factors team at the moment are purely ergonomists, I think I don't think that there are cross trained engineers or anything and I don't think that's entirely satisfactory." (Domain Engineer)

\subsubsection{Costs and resources}

The perception that HF was costly and time consuming was mentioned by respondents a number of times:

"When the organisation tries to avoid using human factors [it] is usually on cost and time scales, sometimes it's sort of internally; we get we get very detailed human factors assessments of work and sometimes that is seen as going over the top for what we need." (Development Engineer)

"I suppose in terms of cost cutting it might be seen as a soft add on." (Safety Team Leader)

Other resource issues, including time, the size and turnover within the team were also seen as having an impact upon integration activities:

"Very few projects would think about human factors at the beginning, and would then try to say 'I've got a human factors integration problem' ... rather than say you can't do everything because one, you don't have the resources, and two, you don't have the money, so what kind of key aspects do they need to focus on?" (Safety Engineer) 
"Overall, the human factors team is quite small. So the people that do leave are the people that have come in and have had two or three years and have gone off to the next thing ... [that] gives the people managing the human factors issues trouble." (Safety Engineer)

\subsection{HFI enablers}

4.4.1 Education and familiarisation courses

Extending the knowledge of human factors amongst engineers, through familiarisation courses for example, was seen as an important mechanism for facilitating HFI within the company:

"Give them a baseline understanding of what HF is, what the benefits of HF are and provide practical examples of how we can help...we're doing that with a HF familiarization course for the designer safety engineers" (Human Factors Team Leader)

"I think more familiarisation training is needed, and to have a basic engineering course, either in earlier employment or as part of their degree course, so they understand the factors involved and the benefits". (Safety Engineer)

Similar courses for the members of the HF team were also mentioned by respondents as filling in the missing gaps in their knowledge of engineering:

"I do think that we should train everybody and give them [the human factors team] awareness training on these things, they don't all have to be practitioners, but engineering is a combination of different things in different elements." (Head of Engineering)

\subsubsection{Organisational factors}

Raising the visibility of HF within the company and adopting a strategy of promoting the value of HF was seen by respondents as a key activity in enabling HFI:

“... hit it with a two pronged approach. If you hit it at the very senior level (sort of the executive vice president of the businesses) and if you can get their buy in, then you have got the buy in of the boss. And then the trick is to also hit it at the lowest level, at the design, engineering level, at the coalface ... then what you have is a sandwich effect. (Human Factors Team Member) 
At the same time persistence and determination to get the message over was recognized as necessary:

"If you don't take a positive attitude to actually keep propagating, promoting human factors [then] that will fly back to the back end again, and the challenge is to actually keep reminding people that they should take this into account." (Safety Engineer)

Part of the issue of raising the visibility of HF was seen an embedding the team within the organization and giving it an acknowledged status within the company:

"We talked about making it a skill group in its own right and that will make it much more visible” (Head of Engineering)

Defining the role of the team and relationship it had to other groups within the company was seen as helping towards greater visibility:

"The challenge that they've got is to actually define what their proper role is, what is their proper scope of activities, where they are going to engage the business or strengths within the company ... basically the challenge is to know what and how HF works in the company." (Safety Engineer)

Actively marketing and selling the value of HF internally was mentioned by respondents as an important mechanism for moving toward greater integration:

"If you can also give them the arguments and say can you afford not to do this? ... that is a good way to get people to buy into human factors and is obviously a good way to improve the relationships between the design people because they will understand better." (Human Factors Team Member)

Finally, improving the timing of exchange of information from the team and enduring effective communication were cited as examples of improvement strategies that could be adopted more widely within the company:

"I think it's mainly communication, being very clear about what we're doing and it's giving a consistent message about what we can do and how we do it. You know trying to get it across to people that it's the not just subjective and that we do have good processes that guide what we do, and kind of align the way in which they work on it as well” (HF Subcontractor Safety Engineer) 


\section{Discussion}

The findings from the interviews with HF and other personnel within the company could be said to be typical of the types of problems we might have expected to be find amongst HF practitioners working within an industrial or commercial context. Comparing our results with previous work carried out on the barriers that exist to HFI demonstrates that many attitudes and perceptions (e.g., that HF is "common sense" and costly) are as prevalent today as they were twenty-five years ago (Meister, 1982a, b; Perrow, 1983, Figure 1). One of the most interesting features of the findings are the range of improvement strategies used by the HF team in order to deal with the barriers to HFI they encountered in their work. The company culture, communication problems and misunderstandings about the functioning and purpose of the team acted together as a hindrance to collaboration with other groups. In the terms of the framework described in section 2 of the paper, the HF team employed a number of strategies and tactics in order to improve perceptions of their performance by other groups in the company, as well as strengthen the degree to which collaborations were viable and sustainable over time (figure 2)

Figure 2 about here

The improvement strategies employed by the HF team in many cases involved them actively trying to break through barriers with other groups. In a number of cases this meant actively trying to adopt the perspective or tap into the "mindset" of engineering groups within the company. In other cases it meant modifying the way in which they presented, or "translated", data to these groups by presenting it in different formats (e.g., presenting quantitative data in a pictorial format). Many of the strategies involved improvements to communications between the groups, as well as actively championing and promoting the value of HF. 
The attempts by the HF team to improve HFI in the company share similarities with other research on the behaviour of newly introduced or minority groups within organisations. Ancona and Caldwell (1990) for example, found that faced with the challenge of raising their profile within a large organisation, new product development teams engaged in a number of boundary management activities. These included setting up roles such as "team ambassador" (e.g., representing the team to other groups, "talking the team up" in order to obtain outside resources) and "team coordinator” (e.g., communicating laterally with other groups, discussing problems with them and gaining feedback on team progress). Within the HF team these types of roles were not set up in such a formal manner, however, they could serve as the basis from which to consider further improvements to HFI within the company.

\subsection{Implications and lessons for HFI}

The study sheds light on an area of investigation that has involved a great deal of discussion and debate over the last few years. Much of this discussion has been driven by methods and tools to narrow the gap between HF personnel and their system development counterparts. The case study we have described provides some further insights into the "softer", more process-oriented aspects of HFI and shows that there are a range of social and organisational strategies that similar HF teams can adopt when attempting to establish better and more productive working relationships with groups from other disciplines. The framework in particular, might serve as a basis with which to plan and evaluate team-based improvement strategies. It could be used for example, to structure discussions centred around gaps in skills and knowledge and other consider options for internal changes to the working of the HF team. Many of the improvement strategies, alongside lessons learnt from other contexts involving boundary management between organisational groups (e.g., Ancona and Caldwell, 1990), might provide other HF teams in similar settings with ideas and interventions designed to improve HFI.

\subsection{Limitations and future work}

The small sample size of our study may well limit the extent to which it generalises to other HFI contexts. Similarly, we examined HFI as it applies within the context of the defence industry, a domain in which HFI shares many similarities with other domains, 
but most likely operates in a different manner. Nevertheless, the fact that our findings chime with those obtained in both the defence and other types of industries leads us to have some confidence in their ability to generalise. Future work need to be carried out in order to evaluate the framework and to explore further types of barriers and improvement strategies that may play a more important role in other contexts. Another line of research that could be explored is tracking the development of changes to attitudes and perceptions, alongside social and organisational developments over a longer period of time. This types of research has yielded important insights within other types of industrial contexts and projects (e.g., software engineering - Walz et al., 1993; Waterson et al., 1997). In our future work we plan to add in a later data collection phase to the study and compare our findings with those described in the present study in order to see how the process of HFI develops over time.

\section{Competing interests}

None

\section{Acknowledgements}

We thank the participants in the study for their time and willingness to take part in the case study, in particular the human factors team leader and the team as a whole, for their cooperation, support and encouragement during the study. The paper has also been greatly improved by the comments on an earlier version by the reviewers for Safety Science.

Word count $=6,052$ 


\section{References}

Ancona, D. and Caldwell, D. 1990. Improving the performance of new product teams. Research Technology Management, 33, 2, 25-9.

Antoni, C. and Hertel, G. 2009. Team processes, their antecedents and consequences: Implications for different types of teamwork. European Journal of Work and Organizational Psychology, 18, 3, 253-266.

Beevis, D., 2003. Ergonomics - costs and benefits revisited. Applied Ergonomics, 34, 491-496.

Bruseberg, A., 2008. Presenting the value of Human Factors Integration: guidance, arguments and evidence. Cognition, Technology and Work, 10, 3, 181-189.

Chapanis, A., 1999. The Chapanis Chronicles: 50 Years of Human Factors Research, Education and Design. Santa Barbara: Aegean Publishing Company.

Cohen, S.G. and Bailey, D.E. 1997. What makes teams work: Group effectiveness research from the shop floor to the executive suite. Journal of Management, 23, 23990.

Cullen, L., 2007. Human factors integration - Bridging the gap between system designers and end-users: a case study. Safety Science, 45, 6211-629.

Crabtree, B.F. and Miller, W.L., 1999. Using codes and code manuals: a template organizing style of interpretation. In B.F. Crabtree and W.L. Miller, (Eds.), Doing Qualitative Research, (2nd edition)._ Newbury Park, California: Sage

Hackman, J.R. 1987, The design of work teams. In J.W. Lorsch (Ed.), Handbook of Organizational Behaviour. Palo Alto, CA: Consulting Psychologists Press.

Kerr, M.P., Knott, D.S., Moss, M.A., Clegg, C.W. and Horton, R.P., 2008. Assessing the value of human factors initiatives. Applied Ergonomics, 39, 305-316. 
King, N., 1998. Template analysis. In G. Symon and C. Cassell (Eds.) Qualitative Methods and Analysis in Organizational Research. London: Sage.

MacLeod, I.S., 2008. Scenario-based requirements capture for human factors integration. Cognition, Technology and Work, 10, 3, 191-198.

Meister, D., 1982a. The role of human factors in systems development. Applied Ergonomics, 13, 2, 281-287.

Meister, D., 1982b. Human factors problems and solutions. Applied Ergonomics, 13, 3, 219-223.

Meister, D., 1999. The History of Human Factors and Ergonomics. Mahwah, NJ: Erlbaum.

Meister, D. and Farr, D.E., 1967. The utilization of human factors information by designers. Human Factors, 9, 71-87.

Miles, M.B. and Huberman, A.M., 1994. Qualitative Data Analysis: An Expanded Sourcebook. Beverly Hills, CA: Sage.

Newman, P. and Tatlock, K., 2004. Barriers and enablers to HFI: final review and recommendations. Systems Engineering and Assessment Ltd (SEA), Report 04/TR/4710.

Newman, P., Bruseberg, A. Lowe, M., Borras, C. and Tatlock, K., 2008. Improving HFI within systems acquisition: methods, tools and future directions. Cognition, Technology and Work, 10, 3, 173-180.

Perrow, C., 1983. The organizational context of human factors engineering. Administrative Science Quarterly, 28, 4, 521-541.

Schein, E.H., 2005. Organizational Culture and Leadership, (3rd Edition), New York: Jossey-Bass 
Sundstrom, E., De Meuse, K. and Futrell, D., 1990. Work teams: applications and effectiveness. American Psychologist, 45, 2, 120-133.

Walz, D.B. Elam, J.J. and Curtis, B., 1993, Inside a software design team: knowledge acquisition, sharing and integration. Communications of the ACM, 36, 10, 63-77.

Waterson, P.E. and Sell, R., 2006. Recurrent themes and developments in the history of the Ergonomics Society. Ergonomics, Vol. 49, 8, 743-799.

Waterson, P.E., Clegg, C.W. and Axtell, C.M., 1997, The dynamics of work organization, knowledge and technology during software development. International Journal of Human Computer Systems, 46, 1, 79-101. 
Table 1: Study participants and experience

\begin{tabular}{ll}
\hline Role & Company experience \\
\hline Human Factors Team Members & \\
HF Team Member & 2 years \\
HF Team Member & 6 years \\
HF Team Leader & 8 years \\
HF Subcontractor Safety Engineer & 4 months \\
Safety Personnel & \\
Safety Case Manager & 11 years \\
Safety Team Leader & 30 years \\
Safety Assessment Subcontractor & 15 years \\
Safety Engineer & 20 years \\
Safety Engineer & 8 years \\
Safety Engineer & 15 years \\
Safety Engineer & 25 years \\
Safety Assessment Subcontractor & 2 years \\
Engineering Personnel & \\
Senior Design Engineer & 9 years \\
Design Engineer & 4 years \\
Technical Engineer & 18 years \\
Development Engineer & 22 years \\
Design Engineer & 3 years \\
Head of Engineering & 35 years \\
Design Engineer & 15 years \\
Domain Engineer & 13 years \\
\hline
\end{tabular}


Table 2: Coding framework

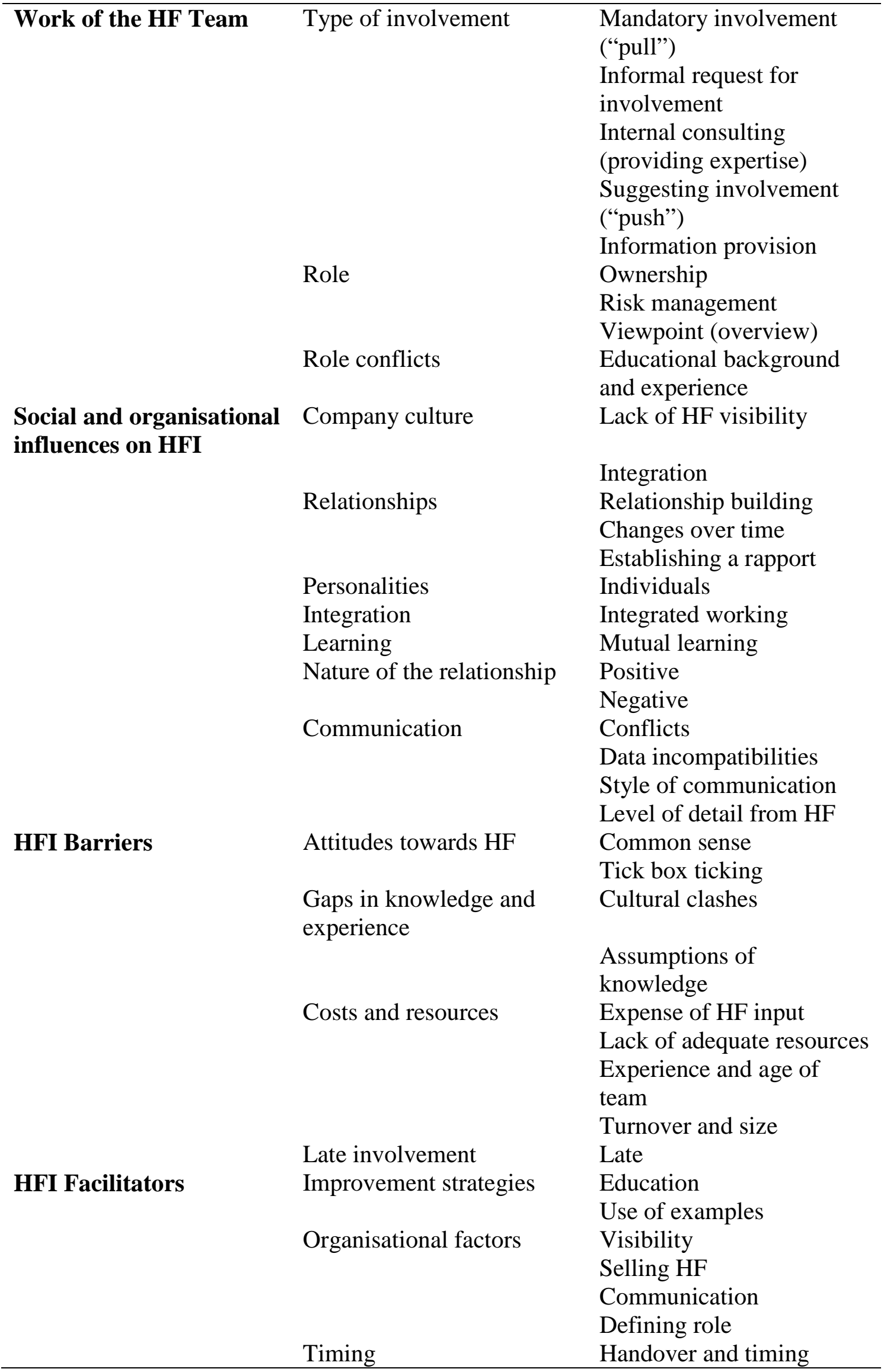


Figure 1: A framework for understanding the social and organisational aspects of HFI

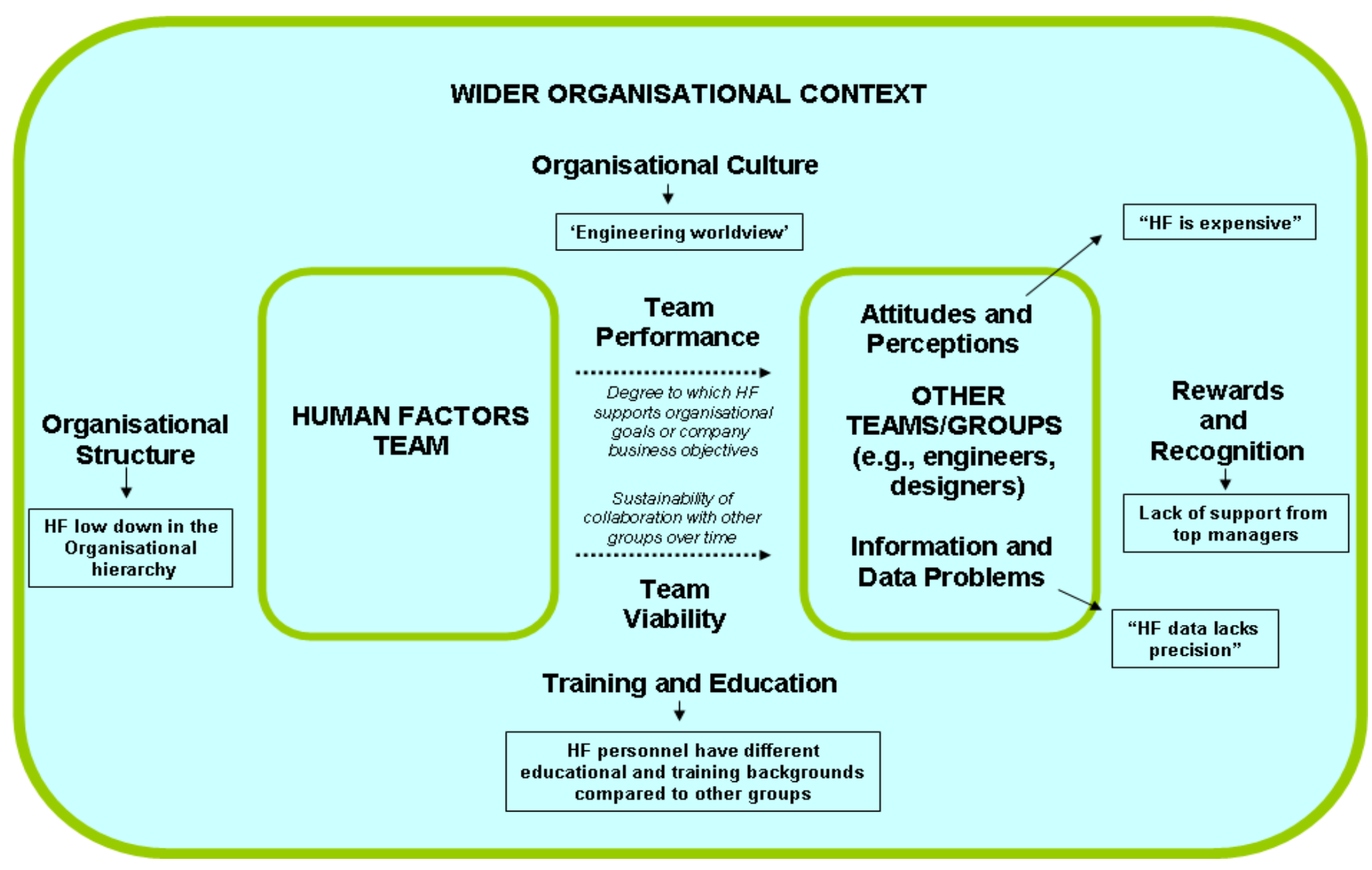


Figure 2: Social and organisational aspects of HFI: Barriers and improvement strategies

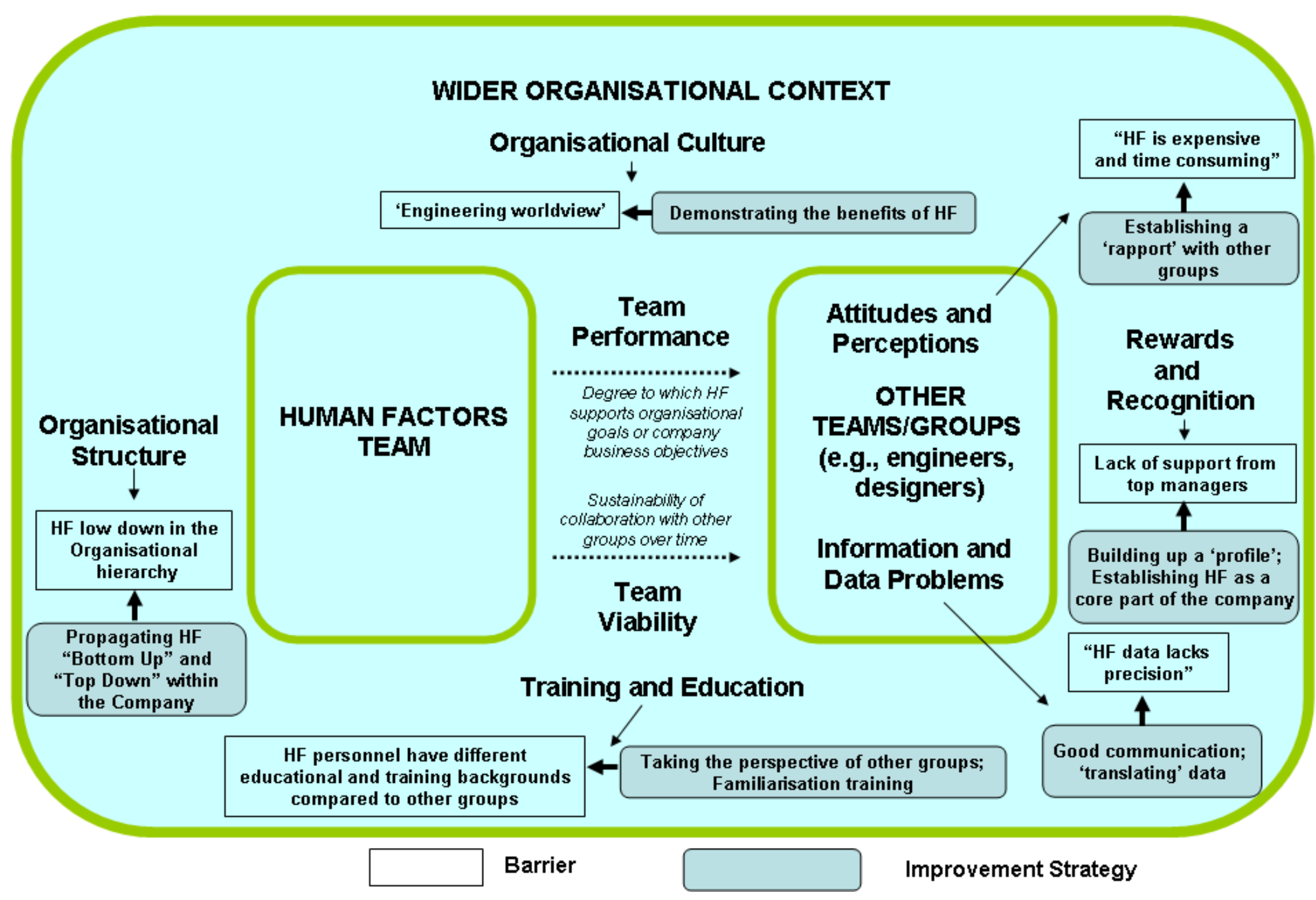

